Purdue University Purdue e-Pubs

2004

\title{
Simulation of Ion Generation and Breakdown in Atmospheric Air
}

W.Zhang

T. S. Fisher

S V. Garimella

Purdue University, sureshg@purdue.edu

Follow this and additional works at: http://docs.lib.purdue.edu/coolingpubs

Zhang, W.; Fisher, T. S.; and Garimella, S V., "Simulation of Ion Generation and Breakdown in Atmospheric Air" (2004). CTRC Research Publications. Paper 292.

http://dx.doi.org/10.1063/1.1806264]

This document has been made available through Purdue e-Pubs, a service of the Purdue University Libraries. Please contact epubs@purdue.edu for additional information. 


\title{
Simulation of ion generation and breakdown in atmospheric air
}

\author{
W. Zhang, T. S. Fisher, ${ }^{\text {a) }}$ and S. V. Garimella \\ School of Mechanical Engineering, Purdue University, West Lafayette, Indiana 47907-2088 \\ and Birck Nanotechnology Center, Purdue University, West Lafayette, Indiana 47907-2088
}

(Received 20 May 2004; accepted 23 August 2004)

\begin{abstract}
Understanding of ion generation in air provides insights to several applications, such as gas sensors, electrohydrodynamic pumping, and air purification. In this paper, ion generation processes in atmospheric air are simulated using a particle-in-cell and Monte Carlo method with emphasis on the prediction of ion generation and breakdown characteristics in microscale gaps. The simulation results are validated through comparison to Townsend's discharge theory and experiments. The significance of each relevant electron-molecule reaction is characterized to improve understanding of ion generation dynamics. Self-sustaining discharge and ionization are predicted under sufficient voltage bias, and the predicted trends of breakdown voltage are similar to those obtained from Paschen's curve. Corrections to Paschen's curve in microscale gaps also are identified and compare well to experiments. Electron field emission produces stable electron current that suggests a controllable ionization device without external electron injection sources. (C) 2004 American Institute of Physics. [DOI: 10.1063/1.1806264]
\end{abstract}

\section{INTRODUCTION}

Ion generation in air is an important process in various applications such as nonmechanical pumping, ${ }^{1}$ isolation breakdown prevention, ${ }^{2}$ and air purification. ${ }^{3}$ However, as the miniaturization of devices continues into micron and submicron regimes, modern applications and concerns have rekindled the interest in the study of microscale gas discharges. Some recently proposed devices such as the gas sensor of Modi et al. ${ }^{4}$ and the electronic-cooling pump of Schlitz et $a l^{5}$ employ small gaps either to enhance ionization current or to reduce threshold voltage. Several recent experiments ${ }^{6,7}$ reveal unique characteristics of microscale discharges as compared to large-scale discharges. Slade and Taylor ${ }^{8}$ indicate that these deviations are related to field emission caused by extremely high electric fields in microscale gaps. In this paper, particle-based numerical simulation methods are employed to study ion generation and breakdown in atmospheric air between closely and widely spaced electrodes. The simulation results show good agreement with prior experimental results. ${ }^{6}$

Several processes occurring within a gaseous medium or at electrodes have been identified since research on gaseous ion generation and ionic breakdown was initiated by Townsend ${ }^{9}$ in the beginning of the last century. The $\alpha$ process involves electron impact ionization. With sufficient energy gained between successive collisions, a free electron can liberate one or more valence electrons from a neutral molecule, resulting in a positive ion and one or more extra free electrons. These free electrons can ionize other molecules and liberate more electrons under favorable conditions. The $\gamma$ process involves secondary electron emission

\footnotetext{
a) Author to whom correspondence should be addressed; Electronic mail: tsfisher@purdue.edu
}

from the cathode surface by various processes such as positive-ion bombardment. An ion strikes the cathode surface with high kinetic energy, causing an electron to be released. The combined effect of the $\alpha$ and $\gamma$ processes is an overexponential growth in the number of charge carriers and the consequent ionic breakdown of the medium. Large-scale gas discharge and breakdown have been studied for many years. Sanders ${ }^{10}$ measured Townsend's first ionization coefficient $(\alpha)$ in air. More recently, Chung et al. ${ }^{11}$ and Smith et al. ${ }^{12}$ have studied gas discharge and breakdown with radio frequency excitation, and ionization coefficients have been reported for other gases and gas mixtures (e.g., helium and tetrakis-dimethylaminoethylene, ${ }^{13}$ argon, and carbon dioxide $^{14}$ ).

Another important cathode process is field emission, which refers to electron emission from a solid surface subjected to a high electric field. As the electrode gap spacing shrinks to the micron range, the local electric field under moderate voltage can exceed a threshold, and electron field emission can become a major electron source near the cathode surface. The anode electron current is enhanced by the addition of field-emitted electrons as well as the electrons that they liberate within the gap. The theory of field emission was first established by Fowler and Nordheim, ${ }^{15}$ and later Good and Müller ${ }^{16}$ provided a comprehensive review of the theory from first principles. To date, most gas discharge studies have considered large gaps with low-pressure conditions relevant to industrial plasma processing ${ }^{17}$ where field emission is negligible. A comprehensive physics-based particle model to simulate both electron field emission and the ensuing ionization processes in the microscale regime has not yet been reported. The present work employs Fowler-Nordheim theory for field emission simulation and applies particle-incell and Monte Carlo (PIC-MC) techniques to simulate ion generation in atmospheric air and thus to predict ionization coefficients, discharge current, and breakdown voltage between two parallel plates. 
TABLE I. Electron-nitrogen reactions included in the present model. $E_{\text {threshold }}$ is the threshold energy in units of $\mathrm{eV}$ and $\sigma_{\max }$ is the maximum cross section in units of $10^{-20} \mathrm{~m}^{2}$.

\begin{tabular}{|c|c|c|c|c|}
\hline Index & Type & $\begin{array}{l}E_{\text {threshold }} \\
(\mathrm{eV})\end{array}$ & $\begin{array}{c}\sigma_{\max } \\
\left(10^{-20} \mathrm{~m}^{2}\right)\end{array}$ & Reference \\
\hline 1 & $e+\mathrm{N}_{2} \rightarrow e+\mathrm{N}_{2}$, elastic & 0.00 & 23.1 & 31 and 41 \\
\hline 2 & $e+\mathrm{N}_{2} \rightarrow e+\mathrm{N}_{2}(r)$, rotational & 0.02 & 3.05 & 41 \\
\hline 3 & $e+\mathrm{N}_{2} \rightarrow e+\mathrm{N}_{2}[v=1(\mathrm{I})]$, vibrational & 0.29 & 0.17 & 41 \\
\hline 4 & $e+\mathrm{N}_{2} \rightarrow e+\mathrm{N}_{2}[v=1(\mathrm{II})]$, vibrational & 1.60 & 5.28 & 41 \\
\hline 5 & $e+\mathrm{N}_{2} \rightarrow e+\mathrm{N}_{2}(v=2)$, vibrational & 1.70 & 3.63 & 41 \\
\hline 6 & $e+\mathrm{N}_{2} \rightarrow e+\mathrm{N}_{2}(v=3)$, vibrational & 1.90 & 2.97 & 41 \\
\hline 7 & $e+\mathrm{N}_{2} \rightarrow e+\mathrm{N}_{2}(v=4)$, vibrational & 2.00 & 2.62 & 41 \\
\hline 8 & $e+\mathrm{N}_{2} \rightarrow e+\mathrm{N}_{2}(v=5)$, vibrational & 2.10 & 1.68 & 41 \\
\hline 9 & $e+\mathrm{N}_{2} \rightarrow e+\mathrm{N}_{2}(v=6)$, vibrational & 2.20 & 1.91 & 41 \\
\hline 10 & $e+\mathrm{N}_{2} \rightarrow e+\mathrm{N}_{2}(v=7)$, vibrational & 2.30 & 1.06 & 41 \\
\hline 11 & $e+\mathrm{N}_{2} \rightarrow e+\mathrm{N}_{2}(v=8)$, vibrational & 2.50 & 0.40 & 41 \\
\hline 12 & $e+\mathrm{N}_{2} \rightarrow e+\mathrm{N}_{2}(A 3, v=0-4)$, electronic & 6.17 & 0.02 & 41 \\
\hline 13 & $e+\mathrm{N}_{2} \rightarrow e+\mathrm{N}_{2}(A 3, v=5-9)$, electronic & 7.00 & 0.07 & 41 \\
\hline 14 & $e+\mathrm{N}_{2} \rightarrow e+\mathrm{N}_{2}(B 3)$, electronic & 7.35 & 0.22 & 41 \\
\hline 15 & $e+\mathrm{N}_{2} \rightarrow e+\mathrm{N}_{2}(W 3)$, electronic & 7.36 & 0.28 & 41 \\
\hline 16 & $e+\mathrm{N}_{2} \rightarrow e+\mathrm{N}_{2}(A 3, v>10)$, electronic & 7.80 & 0.07 & 41 \\
\hline 17 & $e+\mathrm{N}_{2} \rightarrow e+\mathrm{N}_{2}(B-3)$, electronic & 8.16 & 0.09 & 41 \\
\hline 18 & $e+\mathrm{N}_{2} \rightarrow e+\mathrm{N}_{2}(A-1)$, electronic & 8.40 & 0.08 & 41 \\
\hline 19 & $e+\mathrm{N}_{2} \rightarrow e+\mathrm{N}_{2}(A 1)$, electronic & 8.55 & 0.22 & 41 \\
\hline 20 & $e+\mathrm{N}_{2} \rightarrow e+\mathrm{N}_{2}(W 1)$, electronic & 8.89 & 0.09 & 41 \\
\hline 21 & $e+\mathrm{N}_{2} \rightarrow e+\mathrm{N}_{2}(C 3)$, electronic & 11.0 & 1.07 & 41 \\
\hline 22 & $e+\mathrm{N}_{2} \rightarrow e+\mathrm{N}_{2}(E 3)$, electronic & 11.9 & 0.06 & 41 \\
\hline 23 & $e+\mathrm{N}_{2} \rightarrow e+\mathrm{N}_{2}(A 1)$, electronic & 12.3 & 0.04 & 41 \\
\hline 24 & $e+\mathrm{N}_{2} \rightarrow e+\mathrm{N}_{2}$ (sum of singlets), electronic & 13.0 & 1.94 & 41 \\
\hline 25 & $e+\mathrm{N}_{2} \rightarrow e+\mathrm{N}_{2}^{+}$, ionization & 15.6 & 2.15 & 23 \\
\hline 26 & $\begin{array}{c}e+\mathrm{N}_{2} \rightarrow 3 e+\mathrm{N}_{2}^{2+}, \text { ionization } \\
e+\mathrm{N}_{2} \rightarrow 2 e+\mathrm{N}+\mathrm{N}^{+}, \text {ionization }\end{array}$ & 30.0 & 0.76 & 23 \\
\hline 27 & $e+\mathrm{N}_{2} \rightarrow 3 e+\mathrm{N}+\mathrm{N}^{2+}$, ionization & 70.0 & 0.02 & 23 \\
\hline
\end{tabular}

The nonequilibrium nature and small length scales of interest require subcontinuum methods to simulate the relevant physical phenomena. ${ }^{18}$ Continuum fluid mechanics approaches fail at these scales because of the local inequilibrium that exists when the system's characteristic length is of the same order as the carriers' mean free path. (The mean free path of an electron in atmospheric air is $\approx 0.5 \mu \mathrm{m} .{ }^{8}$ ) The particle simulation technique also reveals significant kinetics details, such as average collision energy for each reaction, that typically are not available from continuum methods. The Monte Carlo approach has proven to be successful in simulating rarefied gas flows, such as those found in ion thrusters for space propulsion, ${ }^{19}$ and has been coupled with particlein-cell (PIC) methods successfully to simulate charged particle transport in plasma and radio frequency discharge phenomena. ${ }^{20}$

Crucial inputs for PIC-MC simulation are the scattering cross sections of relevant collisions. The ionization cross sections of oxygen and nitrogen were first reported by Rapp and Englander-Golden, ${ }^{21,22}$ and their work continues to be cited regularly. Straub et al. ${ }^{23}$ recently presented ionization cross sections for different ionization channels that enable differentiation of the contributions of single and multiple ionization events. Itikawa et $a .^{24,25}$ collected cross-section data between electrons and nitrogen and oxygen molecules. The most detailed reviews of electron-molecule elastic and excitation scattering cross sections can be found in recent publications by Morgan ${ }^{26}$ and Brunger and Buckman. ${ }^{27}$

Of particular relevance to the present work are a few recent reports of gas sensors and gas counters. Modi et al. ${ }^{4}$ observed significant changes in breakdown behavior for particular gases $\left(\mathrm{Ar}\right.$ and $\mathrm{NH}_{3}$ ) at volume concentrations above $1 \%$, and a gas sensor based on changes in breakdown voltage caused by trace species is suggested. The gas gain in argonbased gas counters ${ }^{28,29}$ is shown to depend only on the partial pressure of the admixture and exhibits an overexponential relationship with applied voltage. The efficacy of such gas ionization devices will depend on the understanding of molecular kinetics to enable effective filtering of signal noise, and the approach outlined in the present work is capable of including such information and modeling the effects of trace species concentrations.

This paper describes relevant physics of particle-based simulation of ion-generation processes in air within large and small gaps. The model is general and can be used with other gas species and mixtures, although we consider only air in this paper. Ion-generation types and rates are considered as functions of electron kinetic energies and scattering cross sections. Ionization coefficients are calculated and agree well with available experimental data. Breakdown voltages for a variety of gap spacings are obtained and reveal a deviation from the traditional Paschen's curve, and this deviation has 
TABLE II. Electron-oxygen reactions included in the present model. $E_{\text {threshold }}$ is the threshold energy in units of $\mathrm{eV}$ and $\sigma_{\max }$ is the maximum cross section in units of $10^{-20} \mathrm{~m}^{2}$.

\begin{tabular}{|c|c|c|c|c|}
\hline Index & Type & $\begin{array}{l}E_{\text {threshold }} \\
(\mathrm{eV})\end{array}$ & $\begin{array}{c}\sigma_{\max } \\
\left(10^{-20} \mathrm{~m}^{2}\right)\end{array}$ & Reference \\
\hline 28 & $e+\mathrm{O}_{2} \rightarrow e+\mathrm{O}_{2}$, elastic & 0.00 & 9.68 & 33 \\
\hline 29 & $e+\mathrm{O}_{2} \rightarrow e+\mathrm{O}^{-}+\mathrm{O}$, negative ionization & 4.90 & 0.02 & 33 \\
\hline 30 & $e+\mathrm{O}_{2} \rightarrow e+\mathrm{O}_{2}(r)$, rotational & 0.08 & 0.10 & 33 \\
\hline 31 & $e+\mathrm{O}_{2} \rightarrow e+\mathrm{O}_{2}[v=1(\mathrm{I})]$, vibrational & 4.00 & 0.27 & 33 \\
\hline 32 & $e+\mathrm{O}_{2} \rightarrow e+\mathrm{O}_{2}[v=1(\mathrm{II})]$, vibrational & 0.19 & 2.04 & 33 \\
\hline 33 & $e+\mathrm{O}_{2} \rightarrow e+\mathrm{O}_{2}(v=2)$, vibrational & 0.56 & 0.59 & 33 \\
\hline 34 & $e+\mathrm{O}_{2} \rightarrow e+\mathrm{O}_{2}(v=2)$, vibrational & 4.00 & 0.12 & 33 \\
\hline 35 & $e+\mathrm{O}_{2} \rightarrow e+\mathrm{O}_{2}(v=3)$, vibrational & 0.68 & 0.13 & 33 \\
\hline 36 & $e+\mathrm{O}_{2} \rightarrow e+\mathrm{O}_{2}(v=4)$, vibrational & 0.79 & 0.05 & 33 \\
\hline 37 & $e+\mathrm{O}_{2} \rightarrow e+\mathrm{O}_{2}$ (singlet $\Delta$ ), electronic & 0.98 & 0.10 & 33 \\
\hline 38 & $e+\mathrm{O}_{2} \rightarrow e+\mathrm{O}_{2}$ (singlet $\Sigma$ ), electronic & 1.63 & 0.02 & 33 \\
\hline 39 & $e+\mathrm{O}_{2} \rightarrow e+\mathrm{O}_{2}$, electronic & 4.50 & 0.11 & 33 \\
\hline 40 & $e+\mathrm{O}_{2} \rightarrow e+\mathrm{O}_{2}$, electronic & 6.00 & 0.25 & 33 \\
\hline 41 & $e+\mathrm{O}_{2} \rightarrow e+\mathrm{O}_{2}$, electronic & 8.40 & 1.32 & 33 \\
\hline 42 & $e+\mathrm{O}_{2} \rightarrow e+\mathrm{O}_{2}$, electronic & 9.97 & 0.08 & 33 \\
\hline 43 & $e+\mathrm{O}_{2} \rightarrow e+\mathrm{O}_{2}^{+}$, ionization & 12.0 & 1.80 & 23 \\
\hline 44 & $\begin{array}{c}e+\mathrm{O}_{2} \rightarrow 3 e+\mathrm{O}_{2}^{2+}, \text { ionization } \\
e+\mathrm{O}_{2} \rightarrow 2 e+\mathrm{O}+\mathrm{O}^{+}, \text {ionization }\end{array}$ & 18.0 & 1.05 & 23 \\
\hline 45 & $e+\mathrm{O}_{2} \rightarrow 3 e+\mathrm{O}+\mathrm{O}^{2+}$, ionization & 68.0 & 0.03 & 23 \\
\hline
\end{tabular}

been observed in prior experiments. ${ }^{6,7}$ The computations also demonstrate that a stable electron current is possible in small gaps without external excitation sources.

\section{THEORY AND IMPLEMENTATION}

The basic idea of PIC-MC is to use many simulated ("super") particles to represent a much larger number of real particles and to move the particles self-consistently. Simulated particles move subject to applied and space-chargeinduced fields and collide with other particles or boundaries. These collisions are tracked in a Monte Carlo scheme in which the free-flight and collision steps are treated separately. The emitted electrons travel ballistically first, followed by instantaneous binary collisions with target species or boundaries. Meanwhile, the spatial information of charged particles is recorded to calculate the electric field by solving Poisson's equation. Implementation details of the PIC-MC method have been described by Vahedi and Surendra ${ }^{20}$ and Birdsall and Langdon, ${ }^{30}$ and thus are not repeated here.

In the present study air molecules, assumed to be comprised of nitrogen and oxygen only, are regarded as background species and their number densities $n_{t}$ are assumed to be constants. The actual number of possible reaction types between air molecules and electrons is so large that any comprehensive accounting of all would be impractical. The major electron-neutral molecule impact reactions, shown below in Eqs. (1)-(5), are modeled with cross sections available in the literature: ${ }^{23,26,31}$

$$
\begin{aligned}
& \left.e+X_{2} \rightarrow e+X_{2} \text { (elastic, } X=\mathrm{O} \text { or } \mathrm{N}\right), \\
& e+X_{2} \rightarrow e+X_{2}
\end{aligned}
$$

(Excited states) (excitation, $X=\mathrm{O}$ or $\mathrm{N}$ ),

$$
\begin{aligned}
e+X_{2} \rightarrow & 2 e+X_{2}^{+} \text {or } 2 e+X+X^{+} \\
& (\text {single ionization, } X=\mathrm{O} \text { or } \mathrm{N}), \\
e+X_{2} \rightarrow & 3 e+X_{2}^{2+} \text { or } 3 e+X+X^{2+} \\
& (\text { double ionization, } X=\mathrm{O} \text { or } \mathrm{N}), \\
e+\mathrm{O}_{2} \rightarrow & \mathrm{O}+\mathrm{O}^{-} \text {(attachment, oxygen only). }
\end{aligned}
$$

The total number of reactions accounted in this work is 45. Each reaction is assigned an index number from 1 to 45 , and the maximum cross section $\left(\sigma_{\max }\right)$ and threshold energy $\left(E_{\text {threshold }}\right)$ for each reaction are listed in Tables I and II. The complete reaction cross sections can be found in the cited Refs. 23, 26, and 31-33.

Equation (1) involves an elastic collision in which electron and neutral molecules exchange momentum only (reactions 1 and 28). Elastic collisions have the largest cross sections in the lower energy range (shown in Figs. 1 and 2). Although not directly associated with ionization, elastic collisions can alter the electron energy distribution and thus affect the number of electrons possessing energy above the ionization threshold energy. Equation (2) represents many different excitation reactions with different excitation energies. The present simulation includes one rotational excitation reaction each for oxygen and nitrogen (reactions 2 and 30 ), nine vibrational excitation reactions for nitrogen and six for oxygen (reactions 3-11 and 31-36), and thirteen electronic excitation reactions for nitrogen and six for oxygen (reactions 12-24 and 37-42).

Equations (3) and (4) represent single and double ionization reactions in which an incident electron liberates one or two valence electrons from a molecule and forms an ion. Depending on the energy, ionization can be nondissociative or dissociative, i.e., the final ion products can be of either 


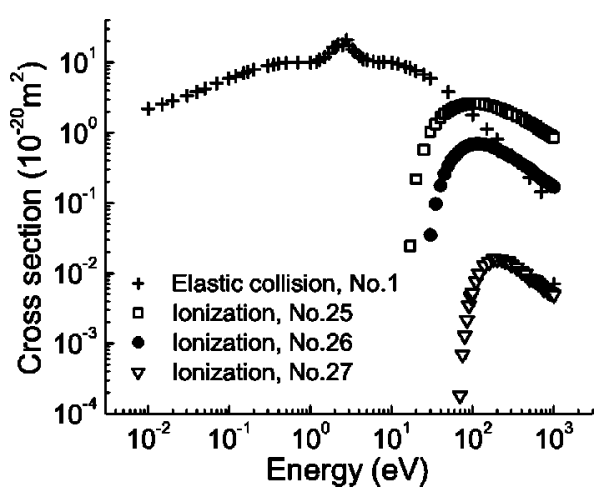

FIG. 1. Cross sections for selected electron-nitrogen molecule reactions (Refs. 23, 31, and 41).

molecular or atomic form. Equation (5) shows that oxygen has a unique reaction in which an electron is attached to a neutral molecule, producing a negative ion (reaction 29). We note that the double molecular ionization and atomic ionization (reactions 26 and 27 for nitrogen; reactions 44 and 45 for oxygen) have lower cross sections than the single molecular ionization (reactions 25 and 43), as shown in Figs. 1 and 2.

The ions are tracked in the Monte Carlo scheme once they are generated. Only elastic and charge-transfer collisions between ions and molecules, the two most probable types of reactions, ${ }^{34}$ are accounted. Because ion-molecule cross sections are not readily available, constant cross sections $\left(1 \times 10^{-18} \mathrm{~m}^{2}\right)$ were assumed with a hard-sphere collision model. ${ }^{20}$

In the analysis of gas discharges, cathode secondary processes play a pivotal role. When sufficiently energetic ions strike the cathode surface, electrons are liberated, accelerated by the electric field, and collide with neutral molecules, thus freeing more electrons and creating more ions. This positive feedback process eventually causes breakdown in large gaps and creates sparks and arcs. To simplify our analysis, we use a constant secondary emission coefficient $\gamma=0.01$, neglecting possible dependence of $\gamma$ on incident ion velocity and cathode surface properties for different electrode materials. ${ }^{35}$

Field emission from the cathode is also included in the present simulations. The phenomenon of field emission from a cold metallic surface occurs as a result of quantum mechanical tunneling of electrons through a surface potential

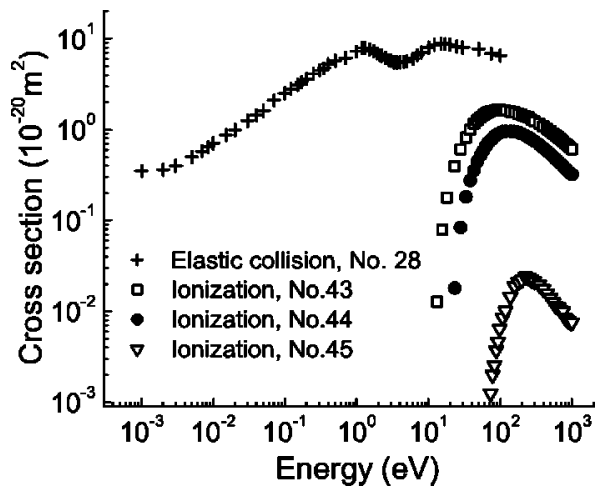

FIG. 2. Cross sections for selected electron-oxygen molecule reactions (Refs. 23, 31, and 33). barrier. From Fowler-Nordheim theory, ${ }^{15}$ the equation relating the surface current density $j\left(\mathrm{~A} \mathrm{~cm}^{-2}\right)$ to the applied electric field $F\left(\mathrm{~V} \mathrm{~cm}^{-1}\right)$ and cathode work function $\phi(\mathrm{eV})$ is

$$
j=\frac{A \beta^{2} F^{2}}{\phi t^{2}(y)} \exp \left\{-\frac{B \phi^{3 / 2} v(y)}{\beta F}\right\} .
$$

In the foregoing equation, $\beta$ represents the enhancement factor for the local surface electric field caused by surface geometry, as compared to the electric field of a perfectly flat surface with the same applied voltage and gap width. The terms $A$ and $B$ are Fowler-Nordheim constants. ${ }^{16}$ The functions $t(y), v(y)$, and $y$ can be approximated as ${ }^{36}$

$$
\begin{aligned}
& y \approx 3.79 \times 10^{-4}(\beta F)^{1 / 2} / \phi, \\
& v(y) \approx 0.95-y^{2}, \\
& t^{2}(y) \approx 1.1 .
\end{aligned}
$$

Once the surface electric field is obtained from the particlein-cell simulation, Eq. (6) is used to determine the fieldemission current density at the cathode. In the computational implementation, each emitted electron is assigned a minimal initial energy, and its position is randomly selected over the cathode surface.

Object-oriented programming has been employed in particle simulations ${ }^{37}$ for many years and offers great coding flexibility and reusability. The present object-oriented code is written to simulate air ionization between two closely spaced parallel electrodes. It is assumed that no other external electron sources exist during the course of simulation, except for field emission and secondary emission. The " $1 \mathrm{~d} 3 \mathrm{v}$ " PIC-MC algorithm by Vahedi and Surendra ${ }^{20}$ was adopted, neglecting the electric field in the lateral directions. Electrons and generated ions are tracked until they reach a boundary. The anode electron current is recorded by counting the electrons arriving at the anode per unit time. Consistency tests indicate that the numerical results do not depend on the discrete time step $\Delta t$ as long as the requirements prescribed in Ref. 20 are satisfied, i.e., electrons move no more than a single grid length in one time step, and the total collision probability for

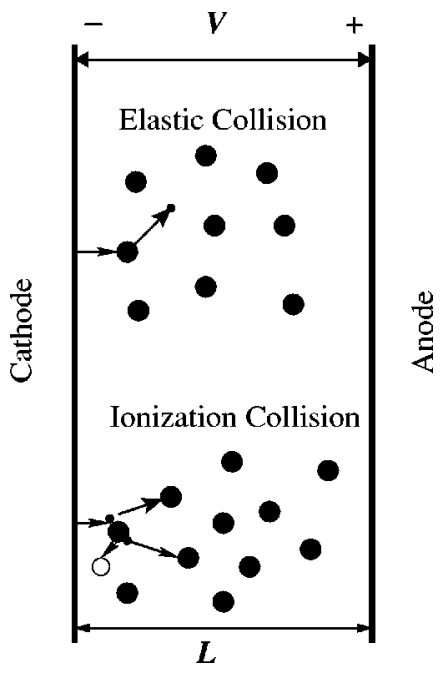

FIG. 3. Schematic diagram of a small gap between two flat-plate electrodes. "O" represents a neutral molecule, "○" represents an ion, and "." represents an electron.

Downloaded 26 Nov 2004 to 128.210.126.199. Redistribution subject to AlP license or copyright, see http://jap.aip.org/jap/copyright.jsp 


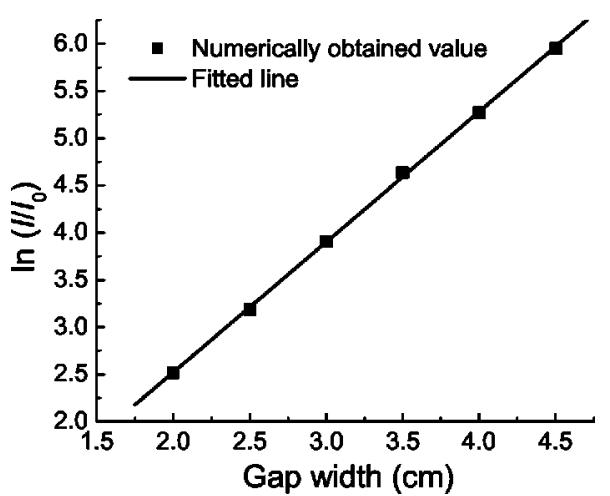

FIG. 4. $\ln \left(I / I_{0}\right)$ as a function of gap width. The slope of the solid straight line is $\alpha$. Electric field is $133 \mathrm{~V} \mathrm{~cm}^{-1}$ and pressure is $0.95 \mathrm{~mm} \mathrm{Hg}$.

any particle in one time step is smaller than 0.095. A schematic diagram of the parallel-electrode problem under consideration is shown in Fig. 3.

\section{RESULTS AND DISCUSSION}

In order to verify the integrity of the present code, Townsend's first ionization coefficient $\alpha$ is calculated for different electric-field-to-pressure ratios. The method described by Nasser ${ }^{38}$ is used to determine $\alpha$. A set of simulations was conducted with equivalent electric fields but different gap widths, assuming a null value for $\gamma$. The natural logarithms of anode-cathode current ratio are then plotted against gap widths. The obtained data can be fit to a straight line whose slope represents the value of $\alpha$. An example result is plotted in Fig. 4 to illustrate this approach, and $\alpha$ is shown to be $1.38 \mathrm{~cm}^{-1}$ for an electric field of $133 \mathrm{~V} \mathrm{~cm}^{-1}$ and a pressure of $0.95 \mathrm{~mm} \mathrm{Hg}$. The $\alpha$ results are compared to the experimental results ${ }^{10}$ in Fig. 5. Close agreement between the simulation and experiments is observed over the entire range of electric fields. Importantly, these results were obtained without the use of any adjustable parameters. We also note that in preliminary simulations including only elastic and single ionization collisions (reactions 1, 25, 28, and 43), the predicted ionization coefficients for these conditions disagreed with experimental values twofold to fourfold, suggesting that inclusion of excitation collisions is critical in describing the physics of breakdown.

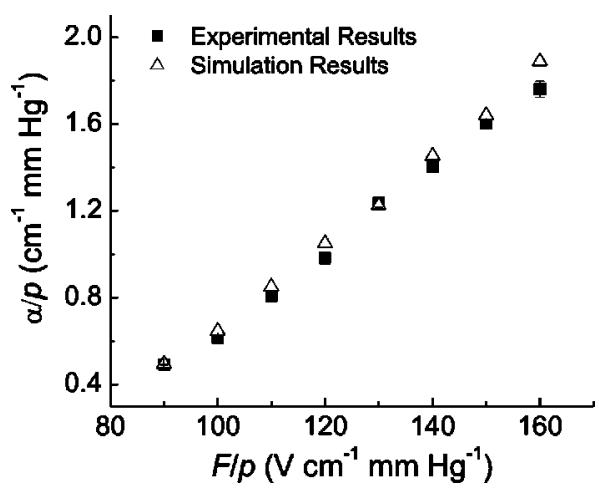

FIG. 5. Comparison between experimental (Ref. 10) and simulation results of Townsend's first ionization coefficient $\alpha$ (divided by pressure) as a function of electric field to pressure ratio $(F / p)$.

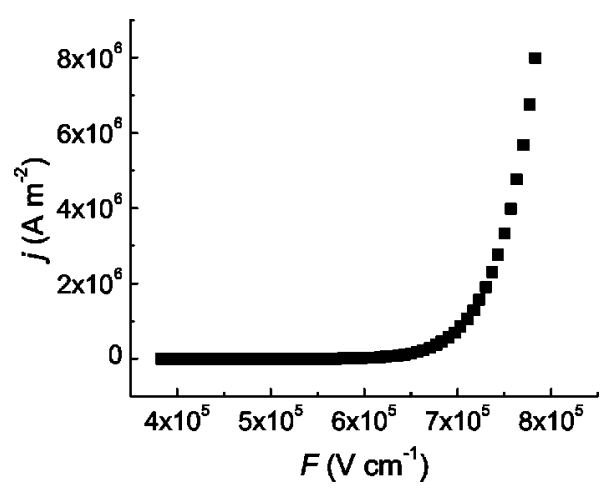

FIG. 6. Field emission current density $(j)$ as a function of applied electric field $(F)$. Field enhancement factor $\beta=55$. Work function $\phi=5.15 \mathrm{eV}$.

Figure 6 shows field emission current density $j$ as a function of applied electric field $F$, based on Eq. (6). A work function $(\phi)$ of $5.15 \mathrm{eV}$ is assumed for nickel, ${ }^{39}$ and the field enhancement factor $(\beta)$ is 55 , which lies in the normal range for metallic materials. ${ }^{16}$ The field emission current density can become comparable to typical discharge current densities at relatively small applied voltages for very small gaps. Consequently, corrections to Paschen's curve for small gaps are required, as discussed later.

With an initial injection of electrons, three different behaviors are considered, namely, "blow-up" (case 1), "stable" (case 2), and "die-off" (case 3), depending on the applied voltage and gap width. The input parameters for each are listed in Table III. The anode electron current serves as the metric to define breakdown. Figure 7 shows the evolution of anode current with time. The anode electron current can increase rapidly, subside, or remain nearly constant depending on the applied voltages and gap widths. If the generation of secondary emitted electrons is large enough to offset the loss of electrons at boundaries, then the electron population grows boundlessly and eventually blows up, as in case 1. On the other hand, if $\gamma$ is too small or the gap is too small, then secondary electrons cannot compensate for electron loss at boundaries, and the electron population dies off as in case 3 . However, for very small gaps, field emission can maintain a sufficient electron population and anode current. The nearly constant anode current in case 2, in which field emission electrons are the major source, suggests that a stable discharge and a steadfast ion source is possible without external excitation. We also note that blow up does not occur in the field-emission-dominated regime considered. To adopt a consistent definition of breakdown in both short gaps and long gaps, breakdown is defined here such that the anode current exceeds $5 \mathrm{~mA}$, following the definition employed by Dhariwal et $a l^{2}$ The active cathode emission area is estimated using the method proposed by Zhu et al. ${ }^{40}$

TABLE III. Input parameters for the three computational cases.

\begin{tabular}{ccccc}
\hline \hline & Case & Applied voltage $(\mathrm{V})$ & Gap $(\mu \mathrm{m})$ & Initial number of electrons \\
\hline 1 & "Blow-up" & 450 & 15 & 100 \\
2 & "Stable" & 160 & 3 & 100 \\
3 & "Die-off" & 250 & 15 & 100 \\
\hline \hline
\end{tabular}




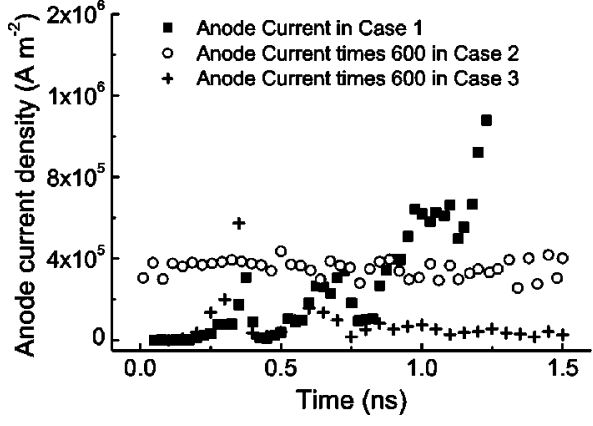

FIG. 7. Anode current vs time in three different cases ("blow-up", "stable", “die-off"). Input parameters are listed in Table III.

Normalized numbers of reaction events, indicating the relative strength for each reaction, are plotted in Fig. 8 for a typical case at time $t=1.03 \mathrm{~ns}$ (applied bias $=450 \mathrm{~V}$, gap $=15 \mu \mathrm{m})$. The plot shows the normalized number of events, corresponding to the number of the reactions divided by the total number of reaction events for nitrogen or oxygen, respectively. Elastic reactions occur most frequently because of their large cross sections. They account for $75 \%$ of all nitrogen reaction events and $81 \%$ of all oxygen events. Aside from elastic collisions, two electronic excitations (reactions 24 and 41) and four ionization events (reactions 25, 26, 43, and 44) occur more frequently than the rest. Among several ionization reactions, single ionization events dominate.

The average electron energy for each reaction also depends on the collision cross section and is plotted in Fig. 9. The average energies for single ionization are $47 \mathrm{eV}$ for both nitrogen and oxygen as they possess similar single ionization cross sections. High thresholds for double ionization and atomic ionization result from obvious difficulties to liberate two electrons or break intermolecular bond in a single collision. Consequently, the average energies for double molecular ionization and single atomic ionization are much higher than those of single ionization. The average energy for ionization collision is approximately $50 \mathrm{eV}$ for both nitrogen and oxygen. This quantity can be used to estimate the mean

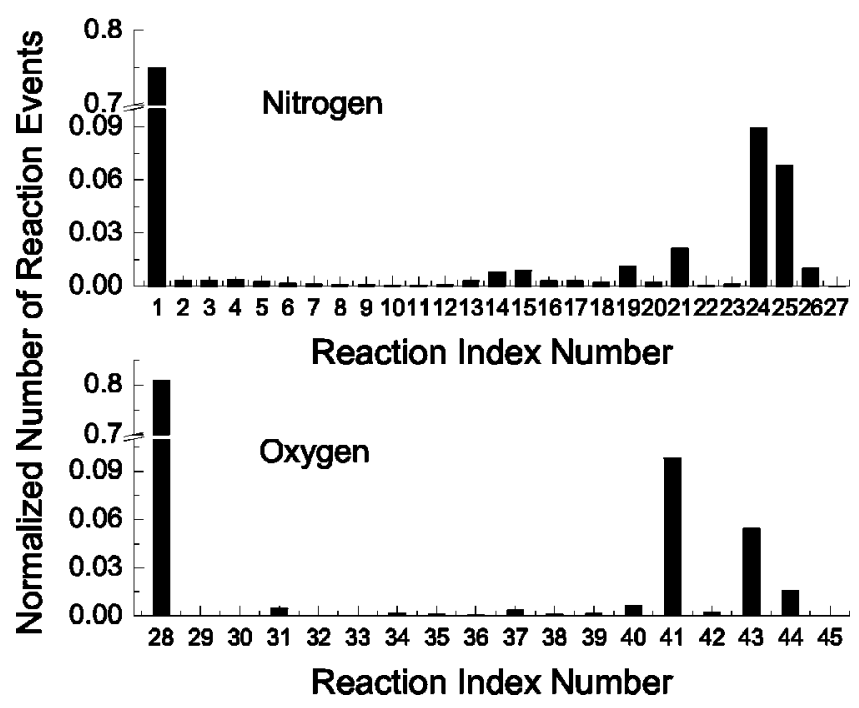

FIG. 8. Normalized number of reaction events for each reaction. Applied bias $=450 \mathrm{~V}$, gap $=15 \mu \mathrm{m}$. The total simulation time is $1.03 \mathrm{~ns}$.

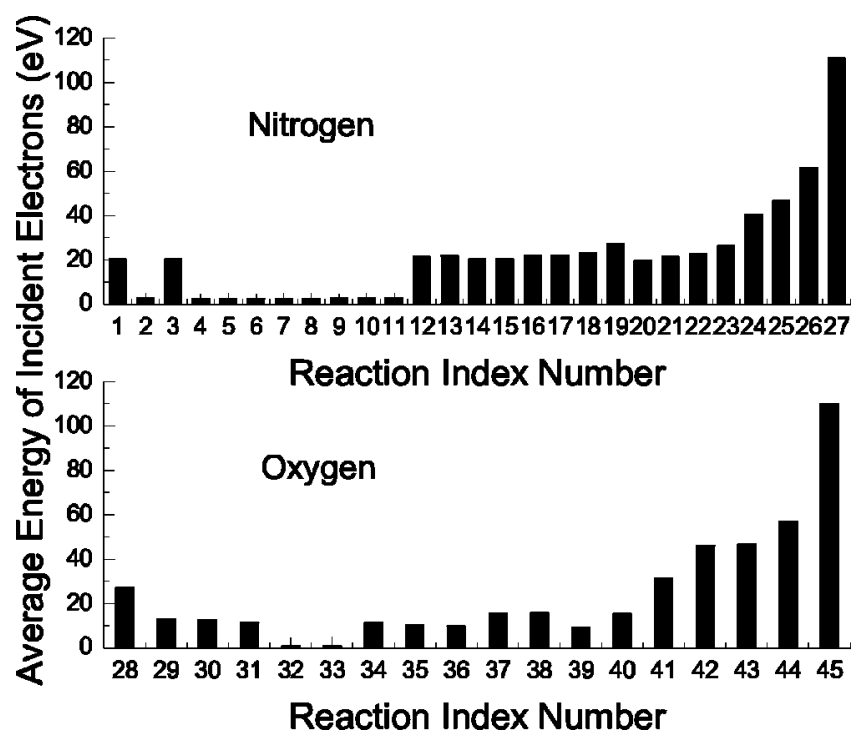

FIG. 9. Average energy of incident electrons for each reaction. Applied bias $=450 \mathrm{~V}$, gap $=15 \mu \mathrm{m}$. The total simulation time is $1.03 \mathrm{~ns}$.

free path for ionization collisions. Assuming that an electron gains energy linearly from the electric field and that the bias is $450 \mathrm{~V}$, it will experience, on average, nine collisions (450/50) during its path length of approximately $15 \mu \mathrm{m}$. Then its mean free path for ionization, by definition, is $15 / 9=1.7 \mu \mathrm{m}$. This approximation is of the expected order of magnitude but should be used with care because mean free path is a strong function of the electron energy distribution, which varies considerably in different scenarios.

Paschen's curve reflects the relationship between breakdown voltage $\left(V_{B}\right)$ and the product of pressure and gap distance. It is used extensively to determine the breakdown limit in electrical design. Paschen's curve ${ }^{38}$ is redrawn in Fig. 10 with an experimental breakdown curve for microscale gaps from Ref. 6 in atmospheric air. Large differences exist between Paschen's curve and experimental results to the left of the minimum breakdown voltage. Slade and Taylor ${ }^{8}$ ascribed this deviation to field emission in an intense electric field.

In order to verify this hypothesis, we conducted a series of numerical simulations and reproduced the breakdown voltage curves for microscale gaps. The results are plotted in Fig. 10, which shows that the simulated data deviate as expected at the lower range of gap width. This result confirms that field emission is the likely reason for the correction to Paschen's curve. When the gap is larger than $7.5 \mu \mathrm{m}$, field emission current becomes negligible and the curve reverts to the typical large-gap trend. Small discrepancies between simulated and experimental results for large gaps are likely caused by two factors. First, the experiments were conducted with curved electrode configurations, which can be approximated by a parallel-plate configuration only for small gaps. Second, the secondary emission process was simplified in the simulation and the energy dependence of $\gamma$ was neglected. Nevertheless, the agreement remains reasonable over the entire range of gap widths. 


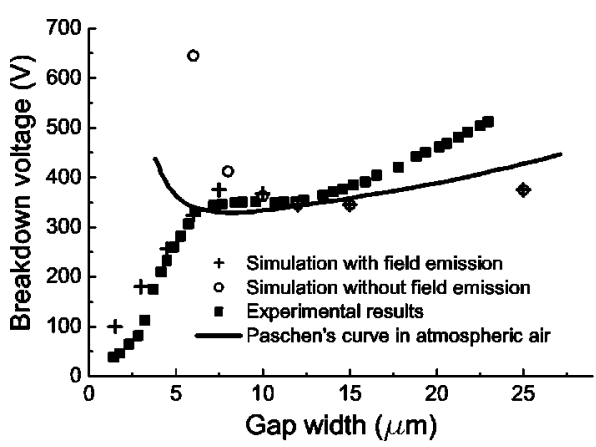

FIG. 10. Simulation-generated Paschen's curve in atmospheric air compared with recent experimental results in microscale gap (Ref. 8) as well as Paschen's curve in atmospheric air (Ref. 38)

\section{CONCLUSIONS}

The PIC-MC method has been used to simulate atmospheric air ionization in microscale gaps, in which field emission can occur. The numerically obtained Townsend's first coefficient agrees well with experimental data. The experimentally observed deviation from Paschen's curve for microscale gaps can be explained by the presence of field emission, and as expected, the field emission effect vanishes for larger gaps. The method can be easily extended to a variety of gases and gas mixtures. For example, the sensitivity and selectivity of the ionization gas sensor proposed by Modi et $a l .{ }^{4}$ can be rigorously predicted using the framework of the current research. Further work will focus on combining the ion-generation simulation with detailed field emission models to provide insights into gas discharges near nanoscale material surfaces and the effects of trace gases.

\section{ACKNOWLEDGMENT}

Support from the Semiconductor Research Corporation and the National Science Foundation for this work is gratefully acknowledged.

${ }^{1}$ J. Darabi, M. Rada, M. Ohadi, and J. Lawler, J. Microelectromech. Syst. 11, 684 (2002).

${ }^{2}$ R. Dhariwal, J. Torres, and M. Desmulliez, IEE Proc.: Sci., Meas. Technol. 147, 261 (2000).

${ }^{3}$ K. Nishikawa and H. Nojima, Jpn. J. Appl. Phys., Part 2 40, L835 (2001).

${ }^{4}$ A. Modi, N. Koratkar, E. Lass, B. Wei, and P. Ajayan, Nature (London) 424, 171 (2003).

${ }^{5}$ D. Schlitz, S. Garimella, and T. Fisher, in ASME International Mechanical
Engineering Congress and RD \& D Exposition, 2003, 41316.

${ }^{6}$ J. Torres and R. Dhariwal, Nanotechnology 10, 102 (1999).

T. Ono, D. Y. Sim, and M. Esashi, J. Micromech. Microeng. 10, 445 (2000).

${ }^{8}$ P. Slade and E. Taylor, IEEE Trans. Compon., Packag. Manuf. Technol., Part A 25, 390 (2002).

${ }^{9}$ J. Townsend, Electricity in Gases (Oxford University Press, New York, 1915).

${ }^{10}$ F. Sanders, Phys. Rev. 44, 1020 (1933).

${ }^{11}$ T. Chung, H. Yoon, T. Kim, and J. Lee, J. Phys. D 29, 1014 (1996).

${ }^{12}$ H. B. Smith, C. Charles, and R. W. Boswell, Phys. Plasmas 10, 875 (2003).

${ }^{13}$ Z. Shuping, P. Bruyndonckx, M. Goldberg, and S. Tavernier, IEEE Trans. Nucl. Sci. 41, 2671 (1994).

${ }^{14}$ G. Auriemma, D. Fidanza, G. Pirozzi, and C. Satriano, Nucl. Instrum. Methods Phys. Res. A 513, 484 (2003).

${ }^{15}$ R. H. Fowler and L. Nordheim, Proc. R. Soc. London, Ser. A 119, 173 (1928).

${ }^{16}$ R. Good and E. Müller, Field Emission, Encyclopedia of Physics Vol. 21, edited by S. Flugge (Springer, Berlin, 1956), pp. 176-231.

${ }^{17}$ A. Rodriguez, W. Morgan, K. Touryan, and W. Moeny, J. Appl. Phys. 70, 2015 (1991)

${ }^{18}$ E. Oran, C. Oh, and B. Cybyk, Annu. Rev. Fluid Mech. 30, 403 (1998)

${ }^{19}$ D. VanGilder, G. Font, and I. Boyd, J. Propul. Power 15, 530 (1999).

${ }^{20}$ V. Vahedi and M. Surendra, Comput. Phys. Commun. 87, 179 (1995).

${ }^{21}$ D. Rapp and P. Englander-Golden, J. Chem. Phys. 43, 1464 (1965).

${ }^{22}$ D. Rapp and P. Englander-Golden, J. Chem. Phys. 43, 1480 (1965).

${ }^{23}$ H. Straub, P. Renault, B. Lindsay, K. Smith, and R. Stebbings, Phys. Rev. A 54, 2146 (1996).

${ }^{24}$ Y. Itikawa et al., J. Phys. Chem. Ref. Data 18, 23 (1989).

${ }^{25}$ Y. Itikawa, M. Hayashi, A. Ichimura, K. Onda, K. Sakimoto, and K. Takayanagi, J. Phys. Chem. Ref. Data 15, 985 (1989).

${ }^{26}$ W. Morgan, Adv. At., Mol., Opt. Phys. 43, 79 (2000).

${ }^{27}$ M. Brunger and S. Buckman, Phys. Rep. 357, 215 (2002).

${ }^{28}$ I. K. Bronić and B. Grosswendt, Radiat. Phys. Chem. 61, 477 (2001).

${ }^{29}$ I. K. Bronić and B. Grosswendt, Nucl. Instrum. Methods Phys. Res. B 168, 437 (2000)

${ }^{30}$ C. Birdsall and A. Langdon, Plasma Physics via Computer Simulation (McGraw-Hill, New York, 1985).

${ }^{31}$ W. Morgan, J. Boeuf, and L. Pitchford, report, 1996 (unpublished), see also http://www.siglo-kinema.com

${ }^{32}$ A. Phelps and S. Lawton, Bull. Am. Phys. Soc. 22, 203 (1977).

${ }^{33}$ S. Lawton and A. Phelps, J. Chem. Phys. 69, 1055 (1978).

${ }^{34}$ A. Phelps, J. Phys. Chem. Ref. Data 20, 557 (1991).

${ }^{35}$ J. R. M. Vaughan, IEEE Trans. Electron Devices 36, 1963 (1989).

${ }^{36}$ C. Spindt, I. Brodie, L. Humphrey, and E. Westerberg, J. Appl. Phys. 47, 5248 (1976).

${ }^{37}$ J. Verboncoeur, A. Langdon, and N. Gladd, Comput. Phys. Commun. 87, 199 (1995).

${ }^{38}$ E. Nasser, Fundamentals of Gaseous Ionization and Plasma Electronics (Wiley-Interscience, New York, 1971).

${ }^{39}$ H. Michaelson, J. Appl. Phys. 48, 4729 (1977).

${ }^{40}$ W. Zhu, C. Bower, O. Zhou, G. Kochanski, and S. Jin, Appl. Phys. Lett. 75, 873 (1999).

${ }^{41}$ A. Phelps and L. Pitchford, Phys. Rev. A 31, 2932 (1985). 